\title{
Effect of Extended Irrigation and Host Resistance on Deoxynivalenol Accumulation in Fusarium-Infected Wheat
}

\author{
M. D. Culler, J. E. Miller-Garvin, and R. Dill-Macky, Department of Plant Pathology, University of Minnesota, St. \\ Paul 55108
}

\begin{abstract}
Culler, M. D., Miller-Garvin, J. E., and Dill-Macky, R. 2007. Effect of extended irrigation and host resistance on deoxynivalenol accumulation in Fusarium-infected wheat. Plant Dis. 91:14641472.

Deoxynivalenol (DON) levels are not easily predicted from visual disease assessment, and it is thought likely that environmental conditions such as temperature and moisture influence DON accumulation. This field study examined the influence of environmental moisture on DON accumulation in Fusarium-infected wheat (Triticum aestivum). The effect of extended mist irrigation applied from inoculation (at anthesis) until harvest was compared with mist irrigation applied from inoculation until disease assessment (at early dough), as is generally applied in screening nurseries used for germplasm selection and cultivar improvement. DON concentrations were quantified in kernels at early dough, hard dough, kernel hard, and maturity. Kernels from plots with extended mist irrigation generally had lower DON concentrations than those from plots where mist irrigation was not applied following disease assessment. DON concentrations tended to decrease from disease assessment until harvest, regardless of the irrigation treatment. DON concentrations in the cultivars moderately resistant to Fusarium head blight were lower than those in the susceptible cultivar. Environmental moisture is an important factor determining the DON content of Fusarium-infected wheat.
\end{abstract}

Fusarium head blight (FHB) is a destructive disease of gramineous crops worldwide. Fusarium graminearum Schwabe (teleomorph: Gibberella zeae (Schwein) Petch) is the Fusarium species most frequently associated with FHB of wheat (Triticum aestivum L.) in the United States and Canada $(14,22,48)$. The disease is initiated when ascospores, released from perithecia on plant residues, infect spikes of wheat at and shortly following anthesis (42). Following infection, $F$. graminearum may rapidly colonize all the tissue of developing spikes (35). High temperatures and extended periods of surface wetness favor infection and colonization. Mist irrigation is often used to promote disease development in screening nurseries used for establishing the response of wheat germplasm to FHB. Irrigation is generally applied between inoculation and disease assessment $(4,47)$ to facilitate perithecial development, ascospore release, and infection $(10,32)$.

Corresponding author: R. Dill-Macky

E-mail: ruthdm@umn.edu

Current address of M. D. Culler: Syngenta Seeds, Inc., Stanton, MN 55018.

M. D. Culler was supported in part by the Minnesota Small Grains Initiative during the completion of his M.S. program at the University of Minnesota.

Accepted for publication 18 June 2007.

doi:10.1094/PDIS-91-11-1464

(c) 2007 The American Phytopathological Society
Wheat is damaged by $F$. graminearum as the mycelium invades the spike and utilizes components of the kernel for fungal growth (3). Kernels infected by Fusarium are small, shriveled, and lightweight; many are lost during harvest (2). Scabby kernels that are retained in the harvested grain reduce quality through reduced test weight. In addition to reducing yield and test weight, $F$. graminearum also may affect grain quality through the production of mycotoxins. Samples of Fusariuminfected wheat harvested from production fields in the United States and Canada routinely test positive for the mycotoxin deoxynivalenol (DON) $(23,36,49,50)$. Other mycotoxins such as diacetoxyscirpenol, HT-2 toxin, T-2 toxin, zearalenone, nivalenol, and acetylated forms of deoxynivalenol (3-acetyl-DON, 15-acetylDON) also may be found in Fusariuminfected wheat $(1,31,36)$. DON is considered the most important mycotoxin produced by $F$. graminearum from a commercial perspective, and is regulated in domestic and international grain markets. DON is a trichothecene mycotoxin with antibiotic, antifungal, and cytostatic activities (17). It inhibits protein and DNA synthesis and has been linked to feed refusal and vomiting in swine and human toxicoses (46).

Studies into the role of DON in infection have demonstrated that it is not essential for the initial infection of spikes. Proctor et al. (33) compared isolates lacking the Tri5 gene (responsible for the production of the enzyme trichodiene synthase and thus the production of DON) to wild-type isolates of $F$. graminearum by inoculation on a susceptible wheat cultivar. FHB severity and incidence data showed that the lack of the Tri5 gene did not affect FHB incidence, but led to lower disease severity compared to the wild-type isolate. This indicated that DON is not essential for infection, although it may be important for the further colonization of host tissue. Similar results were reported by Desjardins et al. (5) and Eudes et al. (8). Studies examining the timing of DON production after infection also support the argument that DON is not essential for infection. Kang and Buchenauer (16) detected DON in wheat tissue at their first sampling, $36 \mathrm{~h}$ after inoculation (h.a.i.) with Fusarium culmorum (W.G. Smith) Saccardo. In their study, DON was found in rachis, phloem, and parenchyma cells above the inoculated spikelet in the absence of hyphae. The authors hypothesized that since DON is water soluble, it was transported to these tissues in the xylem. Savard et al. (37) were able to detect DON at 48 h.a.i. in wheat inoculated with $F$. graminearum. Evans et al. (9) were able to detect DON at 36 h.a.i. in barley inoculated with $F$. graminearum, but could not detect measurable DON in samplings at 0 and 18 h.a.i.

Numerous studies $(11,13,15,19,21)$ have reported lower levels of DON in wheat genotypes with improved FHB resistance compared with susceptible wheat genotypes. Whether this is due to the resistance of the host to initial infection and spread of the fungus in the spike (38) or other modes of resistance has not been proven. Following observations of decreasing DON concentrations in maize from a midseason peak (29) and unequal degradation of labeled DON by resistant and susceptible wheat cultivars (26), mechanisms of resistance conferring the prevention of DON synthesis and the degradation of DON were proposed $(26,28)$.

Researchers have attempted to utilize correlations between visual FHB severity ca. 20 days after infection (d.a.i.) and DON concentrations in harvested grain at ca. 40 d.a.i. in spring wheat growing regions as a means to reduce costs, particularly those for mycotoxin analyses, and increase efficiency of germplasm evaluation $(19,45)$. Although the correlations are frequently significant, $r^{2}$ values can be low and are highly variable from year to year 
$(22,44)$. Reasons for this variability are not well understood, but may be linked to the resistance of the host plants and environmental conditions between infection and harvest.

The effect of environment on DON production, although noted to be a significant factor (24), has not been adequately examined. While DON is known to be produced in large amounts in the early stages of infection (9), the level of DON production declines through host plant maturity $(27,34,39)$. Miller and Young (27) sampled inoculated wheat spikes at intervals that were not explicitly defined, and reported that DON in tissues peaked 6 weeks after inoculation and subsequently declined. Other studies of noninoculated trials in wheat and barley have shown similar results $(34,39)$. While host plant resistance, expressed as DON degradation by plant enzymes, was proposed as an explanation for their results, Miller and Young (27) only examined one cultivar and did not report its resistance level to FHB.

This study was undertaken to examine the influence of environmental moisture on DON accumulation in Fusarium-infected wheat. The specific objective of this study was to identify the impact of mist irrigation and host plant resistance on the production and accumulation of DON by $F$. graminearum in three spring wheat cultivars with varying levels of resistance to FHB.

\section{MATERIALS AND METHODS}

DON accumulation in three spring wheat cultivars, Alsen (moderately resistant to FHB; PVP 200100066), 2375 (moderately resistant to FHB; PVP 8800012), and Wheaton (very susceptible to FHB; PI 469271), was evaluated under two inoculum concentrations in a randomized complete block with a split plot treatment arrangement. Main plot treatments were irrigation treatments, and subplots were the spring wheat cultivars. The experiment was replicated four times and repeated in each of two growing seasons (2002 and 2003). The experiments were planted on the University of Minnesota's Agricultural Experiment Station in Saint Paul, MN.

The wheat cultivars were planted in tworow plots, $3 \mathrm{~m}$ long with $0.3 \mathrm{~m}$ between rows, on 26 April 2002 and 23 April 2003. In 2002, when the plant material was at the third leaf stage (Feekes' growth stage (GS) 1) (51), the field was sprayed with an herbicide tank mixture containing Cheyenne (fenoxaprop-P-ethyl and MCPA, $0.68 \mathrm{~kg}$ a.i./ha; Bayer CropScience, Research Triangle Park, NC) and Harmony Extra (thifensulfuron-methyl and tribenuronmethyl, 15.93 g a.i./ha; DuPont Co., Wilmington, DE) for broadleaf weed control. In 2003, when the plant material was at the eighth leaf stage (GS 4), the trial was treated with Di-Syston 8 (Disulfoton,
0.082 liters a.i./ha; Bayer CropScience Co., Kansas City, MO) for control of several aphid species responsible for transmitting Barley yellow dwarf virus and Bronate (bromoxynil and MCPA, 0.13 liters a.i./ha; Aventis Co., Research Triangle Park, NC) for broadleaf weed control.

Approximately 65 isolates of $F$. graminearum, collected from commercial fields of wheat and barley in 2002 and 2003, which were infected naturally with $F$. graminearum, were used to produce the inoculum. Inoculum production was conducted according to the procedure of Evans et al. (9). Tween 20 (polysorbate; Fisher Biotech, Fair Lawn, NJ) was added to the inoculum at the rate of $0.8 \mathrm{ml}$ per 1 liter of macroconidial suspension. $\mathrm{A} \mathrm{CO}_{2-}$ powered backpack sprayer set at an operating pressure of $276 \mathrm{kPa}$ and fitted with a flat-fan spray tip (TeeJet SS8003, Spraying Systems Co., Wheaton, IL) was used to deliver the inoculum. At anthesis (GS 10.5.1) and 3 days after anthesis, subplots were inoculated with $20 \mathrm{ml}$ of $F$. graminearum inoculum at concentrations of either $2.5 \times 10^{4}$ macroconidia/ml (low concentration treatment) or $1 \times 10^{5}$ macroconidia/ml (high concentration treatment).

Mist irrigation was applied to all plots for 20 min immediately following each inoculation. All inoculations were conducted in the afternoon, with no more than $2 \mathrm{~h}$ from completion of the $20 \mathrm{~min}$ of irrigation to commencement of the overnight irrigation cycle. The mist irrigation ran overnight from 1700 to 0700 hours, with 14 misting periods each night for a total running time of 63 min per day delivering $3.6 \mathrm{~mm}$. The mist irrigation ran in all plots for 15 days in 2002 and 16 days in 2003 following the initial inoculation. Extended mist irrigation treatments were initiated when the plants were at the early dough stage (GS 11.2 (a)). Since early dough and hard dough are both designated by the Feekes' (51) GS number 11.2, we will refer to early dough as GS 11.2 (a) and hard dough as 11.2 (b). Main plots were provided with either extended misting with the irrigation system running at the initial rate $(3.6 \mathrm{~mm} /$ day) (hereafter referred to as extended mist irrigation treatment) or not irrigated from the day of disease assessment until harvest (35 d.a.i.) (hereafter referred to as standard mist irrigation treatment). In extended mist irrigation treatments, the irrigation systems ran for an additional 16 days in 2002 (total 31 days) and an additional 17 days (total 32 days) in 2003. Main plots were spaced at least $9 \mathrm{~m}$ apart to avoid interplot interference.

FHB severity was assessed for each subplot 16 d.a.i. (GS 11.2 (a)) in 2002 and 17 d.a.i. (GS 11.2 (a)) in 2003. FHB severity was calculated by dividing the number of visually symptomatic spikelets by the total number of spikelets for each of 20 arbitrarily selected spikes per subplot and multiplying the resulting mean value of the 20 spikes by 100 .

In 2002, 60 FHB-infected spikes from the low inoculum concentration with less than $50 \%$ severity were selected arbitrarily from each subplot and tagged with a piece of colored tape around the peduncle. Sixty spikes per subplot from the high inoculum concentration with greater than $50 \%$ visual infection were likewise selected and tagged. Concurrently, 10 spikes fitting the selection parameters from each subplot were sampled. Subsequent samples of 10 spikes per subplot were collected from tagged spikes at hard dough (GS 11.2 (b)), kernel hard (GS 11.3), and harvest ripe (GS 11.4). The spike samples were placed in plastic bags and stored at $-20^{\circ} \mathrm{C}$. The spikes from each sample were dissected and the kernels from each sample placed in 50-ml polypropylene centrifuge tubes (Corning Inc., Corning, NY) and returned to $-20^{\circ} \mathrm{C}$. DON analysis was conducted on the kernels.

In 2003, 10 spikes were collected arbitrarily from each subplot at the end of anthesis (GS 10.5.3) and at early milk (GS 11.1). Seventeen d.a.i. (early dough, GS 11.2 (a)), 60 FHB-infected spikes in each subplot from the low inoculum concentration treatment with one visually infected spikelet were selected and tagged as previously described. Sixty FHB-infected spikes with four or more visually infected spikelets in each subplot from the high inoculum concentration treatment were selected similarly and tagged. Concurrently, 10 spikes fitting the selection parameters were collected from each subplot. Subsequent samples of 10 tagged spikes were collected at hard dough (GS 11.2 (b)), kernel hard (GS 11.3), and harvest ripe (GS 11.4). All samples collected were stored in plastic bags at $-20^{\circ} \mathrm{C}$. For the samples collected at anthesis (GS 10.5.1) and early milk (GS 11.1), all the embryos from an arbitrarily selected group of three adjacent spikelets per spike were dissected from the spike and dried at $60^{\circ} \mathrm{C}$ for $16 \mathrm{~h}$. In samples collected after the early milk stage, spikes were dried at $60^{\circ} \mathrm{C}$ for $16 \mathrm{~h}$, and all kernels were separated from all other spike material. The embryos or kernels were analyzed for DON.

In 2003, visually scabby kernels (VSK) were determined for the grain harvested from the subplots. The subplots were harvested mechanically at maturity, and the grain was dried under forced air at $95^{\circ} \mathrm{C}$ for $48 \mathrm{~h}$. The dried grain samples were cleaned using a University of Minnesotadesigned forced-air seed cleaner, and then a 25-g sample was taken using a Boerner divider (Seedburo, Chicago, IL) and cleaned further by hand. The percentage of VSK for each sample was determined by comparing the seed samples to standards according to the procedures developed by Jones and Mirocha (15). In 2002, the grain was damaged severely by $F$. graminearum, 
and VSK analysis was not possible as a majority of the samples exceeded $50 \%$, which was the maximum value on the VSK scale.

In both years, precipitation data were collected on the St. Paul, MN campus at a location within $750 \mathrm{~m}$ of the field trials. Rainfall totals from inoculation to harvest in 2002 and 2003 were similar, at $229 \mathrm{~mm}$ in 2002 and $197 \mathrm{~mm}$ in 2003 . There were 14 rainfall events in 2002 and 17 rainfall events in 2003 (Fig. 1). Despite similar rainfall totals and the number of precipitation events, the distribution of rainfall over the growing season in 2002 was quite different than in 2003. In 2003, rainfall events greater than $10 \mathrm{~mm}$ mainly occurred within 20 days after anthesis. In 2002, rainfall events were more evenly distributed between anthesis and harvest, although rainfall events were slightly more frequent toward harvest.

DON was quantified using combination gas chromatography and mass spectrometry (GC-MS) as described by Mirocha et al. (30). Briefly, DON was extracted from the samples (2 to $6 \mathrm{~g}$ in 2002 and $4 \mathrm{~g}$ in
$2003)$ in $40 \mathrm{ml}$ (2002) and $16 \mathrm{ml}$ (2003) of acetonitrile-water $(84: 16 \mathrm{vol} / \mathrm{vol})$ and placed on a rotary shaker for $24 \mathrm{~h}$ (2002)

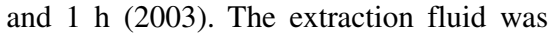
then passed through a specially designed column consisting of one part $\mathrm{C}_{18}$ packing material to three parts aluminum oxide neutral in a 1-ml tuberculin syringe (43). Two 1-ml aliquots of the extraction fluid were removed, and each was transferred to 2-ml screw top vials and evaporated to dryness with nitrogen. The DON in the extract was derivatized to its trimethylsilyl ether derivative for GC-MS analysis (30). A 1- $\mu$ l sample was injected into a combination gas chromatograph and mass spectrometer (Model QP2010; Shimadzu Ltd., Kyoto, Japan). The presence of DON was verified positively based on retention times and selected ion monitoring $(\mathrm{m} / \mathrm{z})$. DON concentrations were determined by comparing peak areas to known standards included at regular intervals and adjusted according to individual sample weights.

FHB severity data were analyzed as a randomized complete block design with eight replicates, using the general ANOVA/
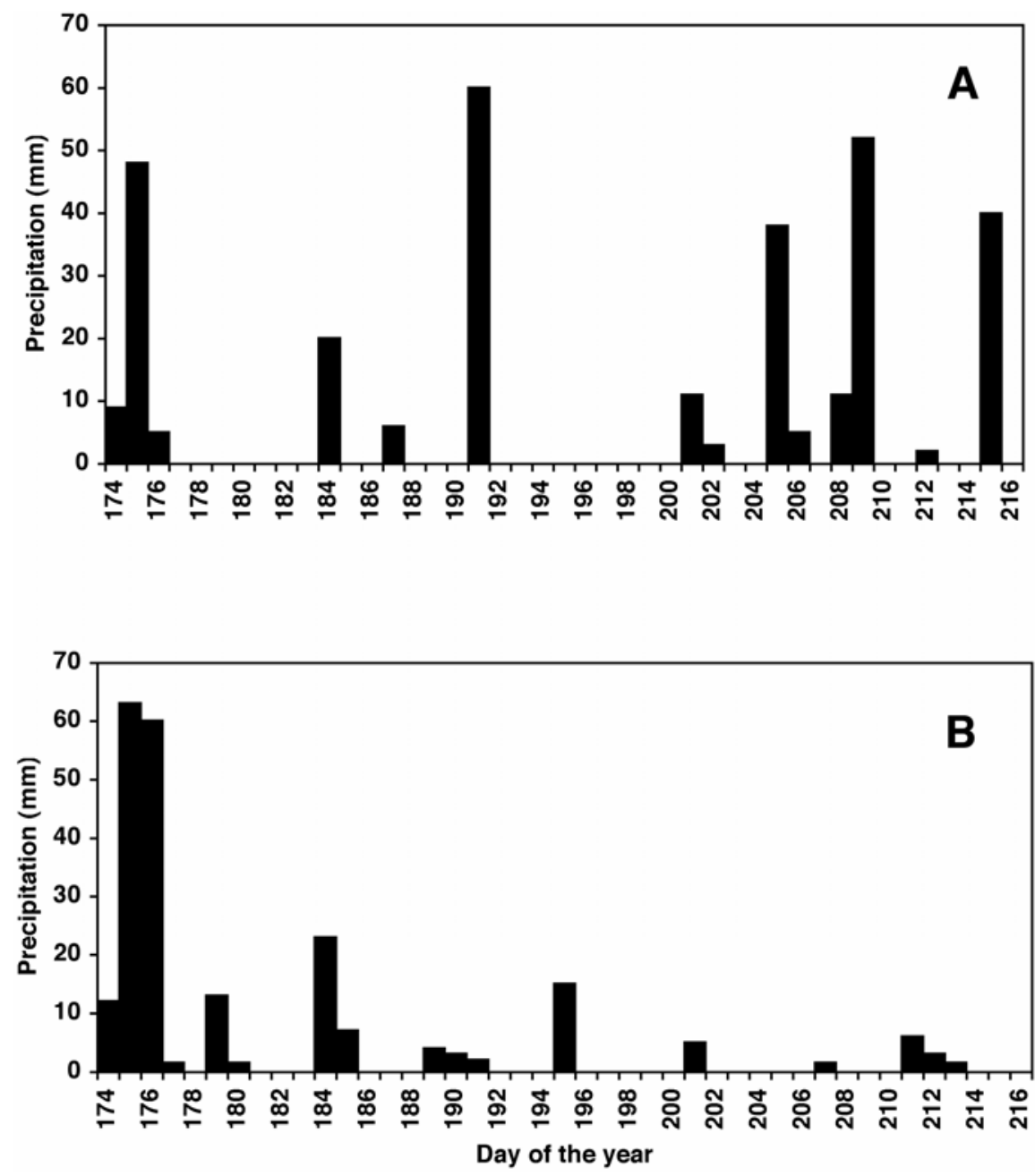

Fig. 1. Total daily precipitation (mm) from inoculation to harvest in 2002 (A) and 2003 (B) at St. Paul, MN. Dates of inoculation for 2002 were 179 for wheat cultivars Alsen and 2375, and 181 for cultivar Wheaton, and date of harvest for 2002 was 216. Dates of inoculation for 2003 were 174 for 'Alsen' and '2375', and 175 for 'Wheaton', and date of harvest was 212.
ANCOVA procedure in Statistix 7 (Analytical Software, 2000, Tallahassee, FL) because the data were collected prior to the initiation of irrigation treatments. VSK and DON data were analyzed as a randomized complete block with a split plot treatment arrangement using PROC GLM of SAS (SAS Institute, Research Triangle Park, NC). DON data were analyzed for each sample date separately. Least significant differences (LSD) for comparisons between main plot means (irrigation treatments) for the same subplot (cultivar) were computed according to the methods of Gomez and Gomez (12). Data were considered significantly different at $P<0.05$. Correlation analysis was conducted on means for FHB, DON, and VSK using Microsoft Excel 2004 version 11.3, which calculates Pearson's correlation coefficient.

\section{RESULTS}

FHB severity. FHB severity was higher in 2002 than in 2003 for 'Alsen', '2375', and 'Wheaton' under both low and high inoculum concentrations (Table 1). In both years, under the high inoculum concentration, FHB severities for 'Wheaton' were significantly higher than for either 'Alsen' or '2375'. The FHB severities for 'Alsen' and ' 2375 ' were similar, with those for ' 2375 ' being slightly lower than 'Alsen' in both years. There were no significant differences among the wheat cultivars tested in either year under the low inoculum concentration.

VSK. VSK data were only collected in 2003. For all cultivars and both low and high inoculum concentrations, VSK values were higher under extended mist irrigation than under standard mist irrigation (Table 2 ), although this difference was only statistically significant for ' 2375 ' under the high inoculum concentration treatment.

DON concentration in developing and mature kernels. DON concentration data for 2002 and 2003 were not combined for analysis because the DON concentrations differed dramatically between the years, as did the FHB severities. Further, because the effect of inoculum concentration on generating differing levels of FHB is known (7), data for the two inoculum concentrations were treated as distinct disease situations and were used to examine DON accumulation and cultivar responses separately.

Low inoculum concentration (Fig. 2). In 2002, the profile of DON concentrations over the growth stages for 'Alsen', '2375', and 'Wheaton' differed between irrigation treatments. DON concentrations tended to decrease from early dough (GS 11.2 (a)) to maturity (GS 11.4) under extended mist irrigation for '2375' and 'Wheaton'. DON concentrations for 'Alsen' in the extended mist irrigation treatment were more constant, and did not appear to follow an obvious pattern. There was no obvious pat- 
tern to the profile of DON concentrations under the standard mist irrigation treatment for any of the cultivars. The only significant difference between irrigation treatments was for 'Wheaton' at kernel hard and maturity. The DON concentration under extended mist irrigation was significantly lower $(P<0.05)$ than under standard irrigation.

In 2003, DON concentrations were determined in samples collected at the end of anthesis (GS 10.5.3) and early milk (GS 11.1 ), in addition to the growth stages sampled in 2002. No DON was detected at the end of anthesis. DON concentrations ranged from undetectable to $1.03 \mathrm{ppm}$ at early milk (Fig. 2). The profiles of DON concentrations over the remaining sampling dates were similar for both mist irrigation treatments for 'Alsen' and '2375'. For 'Alsen', DON concentrations peaked around hard dough (GS 11.2 (b)), whereas those of ' 2375 ' peaked at kernel hard (GS 11.3). DON concentrations for both 'Alsen' and ' 2375 ' had decreased from the peak values by maturity for both irrigation treatments. The DON profiles for 'Wheaton' were somewhat different. Under the standard irrigation treatment, DON concentration peaked at hard dough and then decreased through maturity, but under the extended mist irrigation treatment, DON concentration was similarly high at hard dough and kernel hard and then decreased at maturity. Only for 'Wheaton' at hard dough was the difference between irrigation treatments significant, where DON concentrations under the extended mist irrigation were significantly lower $(P<$ $0.05)$ than under standard irrigation.

In both years, DON concentrations generally were lower under extended mist irrigation for all cultivars at hard dough, kernel hard, and maturity. Although these differences were generally not significant $(P>0.05)$, this trend was observed consistently.

High inoculum concentration (Fig. 3). In 2002, DON concentrations decreased with maturity for all cultivars under both irrigation treatments. DON concentrations in the standard mist irrigation treatment were highest at early dough (GS 11.2 (a)) for each cultivar and were lowest at maturity (GS 11.4). Similarly, under extended mist irrigation, DON concentrations were highest at early dough, but were generally lowest at kernel hard (GS 11.3) and then remained steady or increased again somewhat by GS 11.4. DON concentrations were significantly lower $(P<0.05)$ for all cultivars under extended mist irrigation than under standard mist irrigation at kernel hard. At maturity, DON concentrations were significantly lower under extended mist irrigation for 'Alsen' and 'Wheaton' than under standard mist irrigation. Differences in DON concentrations between irrigation treatments were not significant $(P>0.05)$ for ' 2375 ' at maturity.
In 2003, DON concentrations were determined in samples collected at the end of anthesis and early milk, in addition to the growth stages sampled in 2002. No DON was detected at the end of anthesis, and DON concentrations ranged from 0.40 to $1.48 \mathrm{ppm}$ at early milk (Fig. 3). DON concentration profiles under both irrigation treatments in 2003 for 'Alsen' and '2375' were similar. DON concentrations appear to have peaked between early dough and kernel hard under both irrigation treatments for 'Alsen' and '2375', and in all cases, DON concentrations decreased at subsequent growth stages. Profiles of DON concentrations in 'Wheaton', the FHB susceptible cultivar, were different for the two irrigation treatments. DON concentrations decreased at each growth stage sampled under extended mist irrigation, but increased at each growth stage under standard mist irrigation. Differences in DON concentrations between the irrigation treatments were only significant for Wheaton at maturity, with DON concentrations being significantly lower $(P<0.05)$ under extended mist irrigation than under standard mist irrigation.

In both years, DON concentrations were lower under extended mist irrigation for all cultivars at hard dough, kernel hard, and maturity, although many of these differences were not significant.

In our study, we found multiple instances where the correlation coefficients between FHB severity and DON concentrations were significant. In 2002, correlations between FHB severity and DON were significant at early dough $(r=0.97$,

Table 1. Fusarium head blight (FHB) severity for wheat cultivars Alsen, 2375, and Wheaton inoculated with a mixture of isolates of Fusarium graminearum (low inoculum concentration, $2.5 \times 10^{4}$ macroconidia/ml or high, $1 \times 10^{5}$ macroconidia/ml) in St. Paul, MN in 2002 and $2003^{\mathrm{y}}$

\begin{tabular}{|c|c|c|c|c|}
\hline \multirow[b]{3}{*}{ Cultivar } & \multicolumn{4}{|c|}{ FHB severity (\%) } \\
\hline & \multicolumn{2}{|c|}{ Low concentration } & \multicolumn{2}{|c|}{ High concentration } \\
\hline & 2002 & 2003 & 2002 & 2003 \\
\hline Alsen & $26.9 \mathrm{a}^{\mathrm{z}}$ & $10.0 \mathrm{a}$ & $51.8 \mathrm{a}$ & $15.3 \mathrm{a}$ \\
\hline 2375 & $29.4 \mathrm{a}$ & $9.5 \mathrm{a}$ & $50.6 \mathrm{a}$ & $13.4 \mathrm{a}$ \\
\hline Wheaton & $35.6 \mathrm{a}$ & $11.5 \mathrm{a}$ & $70.1 \mathrm{~b}$ & $25.4 \mathrm{~b}$ \\
\hline
\end{tabular}

y Values represent the mean of eight replicates.

${ }^{z}$ Values within columns followed by the same letter are not statistically different at $P>0.05$.

Table 2. Visually scabby kernels (VSK) for wheat cultivars Alsen, 2375, and Wheaton in harvested grain collected at maturity (growth stage 11.4) (51) following inoculation with a mixture of isolates of Fusarium graminearum (low inoculum concentration, $2.5 \times 10^{4}$ macroconidia/ml or high, $1 \times 10^{5}$ macroconidia/ml) for two irrigation treatments following disease assessment ${ }^{y}$

\begin{tabular}{llcc}
\hline & & \multicolumn{2}{c}{ VSK (\%) at maturity } \\
\cline { 3 - 4 } Cultivar & Irrigation treatment & Low concentration & High concentration \\
\hline \multirow{2}{*}{ Alsen } & Extended mist irrigation & 9.6 & 16.8 \\
& Standard mist irrigation & 7.0 & 12.4 \\
& Extended mist irrigation & 19.4 & $34.4^{\mathrm{z}}$ \\
\multirow{2}{*}{ Wheaton } & Standard mist irrigation & 9.4 & $10.4^{\mathrm{z}}$ \\
& Extended mist irrigation & 56.3 & 68.8 \\
& Standard mist irrigation & 45.6 & 62.5 \\
\hline
\end{tabular}

${ }^{y}$ Values represent the means of four replicates from St. Paul, MN in 2003.

${ }^{\mathrm{z}}$ Indicates paired (within a cultivar and single inoculum concentration) treatment means which are statistically different at $P<0.05$. , significant at $P=0.01$ ), kernel hard maturity $(r=0.68$, significant at $P=0.01)$; 2003 the correlations between FHB dough $(r=0.98$, significant at $P=$ $P=0.01)$, kernel hard $(r=0.87$, significant $=0.01)$, and maturity $(r=0.86$, sigtions were observed for DON at early , which is when we assessed FHB in the environment following the affected the cultivars differentially and observed at later growth stages. We found gnificant correlation between VSK and $=0.05)$; however, this correlation was wer than we might have expected since formed on the same grain sample used to VSK, and VSK was thus grain damage than field FHB severity, examination of hisual glumes) several weeks earlier. We found a correlation between FHB severity and

\section{TSUSSION}

indicated that moisture conditions between disease assessment at hard dough and har- significant at $P=0.01)$, hard dough $(r=$ tion treatments were imposed. Presumably, 
vest influenced DON accumulation in Fusarium-infected kernels. DON concentrations at harvest were lower under extended mist irrigation than under standard mist irrigation. The impact of extending the days of mist irrigation was most apparent for the FHB susceptible cultivar Wheaton, and less so for the moderately resistant cultivars Alsen and 2375. The trend for decreasing DON concentrations over the sampling period under extended mist irrigation was not expected. Mist irrigation is applied frequently to research plots following inoculation to facilitate the infection of wheat by $F$. graminearum and the development of FHB (6). Extended irrigation was expected to favor disease development and lead to increased Fusarium colonization, and thus greater DON accumulation in grain between infection and harvest. The application of extended mist irrigation may have promoted the spread of $F$. graminearum readily through host tissue without promoting the accumulation of DON.

Free moisture on the spikes, such as that delivered by an irrigation system, may have led directly to the decreases in DON concentrations. DON is a water-soluble compound, and it has been reported to be transported in the xylem vessels to por-
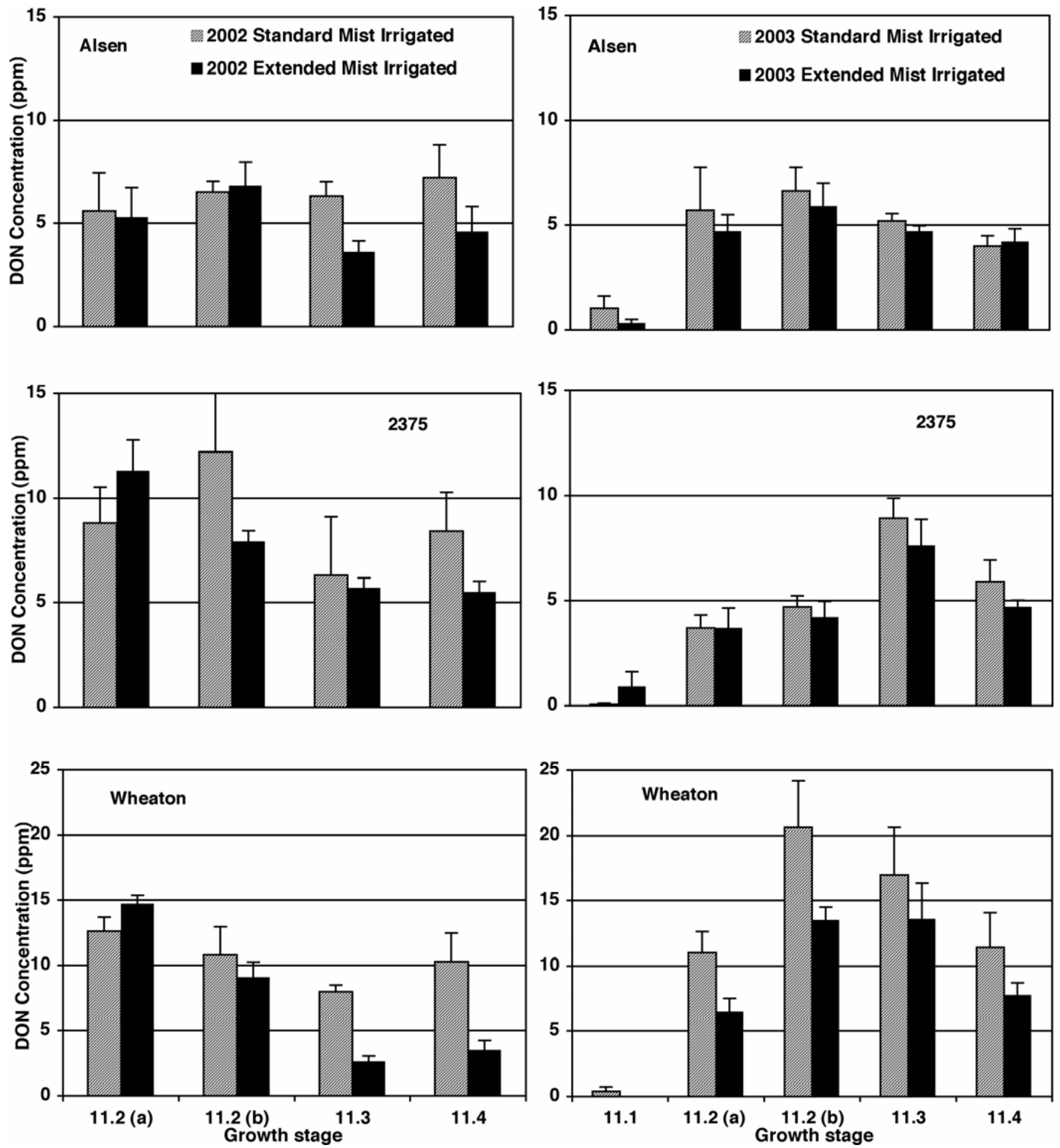

Fig. 2. Deoxynivalenol (DON) concentration in kernels of wheat cultivars Alsen, 2375, and Wheaton collected at growth stages 11.2 (a) (early dough), 11.2 (b) (hard dough), 11.3 (kernel hard), and 11.4 (harvest ripe/maturity) in 2002, and 11.1 (early milk), 11.2 (a), 11.2 (b), 11.3, and 11.4 in 2003, following inoculation with a mixture of isolates of Fusarium graminearum (low inoculation concentration, $2.5 \times 10^{4}$ macroconidia/ml) in plots treated either with extended mist irrigation $(3.6 \mathrm{~mm} /$ day) or standard mist irrigation following disease assessment at St. Paul, MN. Error bars indicate standard errors of means. 
tions of the spike not colonized by $F$. graminearum (16). It is therefore possible that free water on the spikes was able to leach DON from the developing kernels.

Decreases in DON concentrations from early dough to maturity also occurred in samples collected under the standard irri- gation treatment, as used in most inoculated and mist irrigated screening nurseries where irrigation systems are shut off when visual disease assessments are taken. Leaching may not be the sole explanation for the decrease in DON concentration as grain matures. Similar decreases in DON concentration with maturity have been seen in previous studies. Miller and Young (27) reported a decline in DON concentration in sampled spikes in an inoculated field experiment. In their study, a field plot was inoculated at anthesis and spike samples were taken at approximate weekly
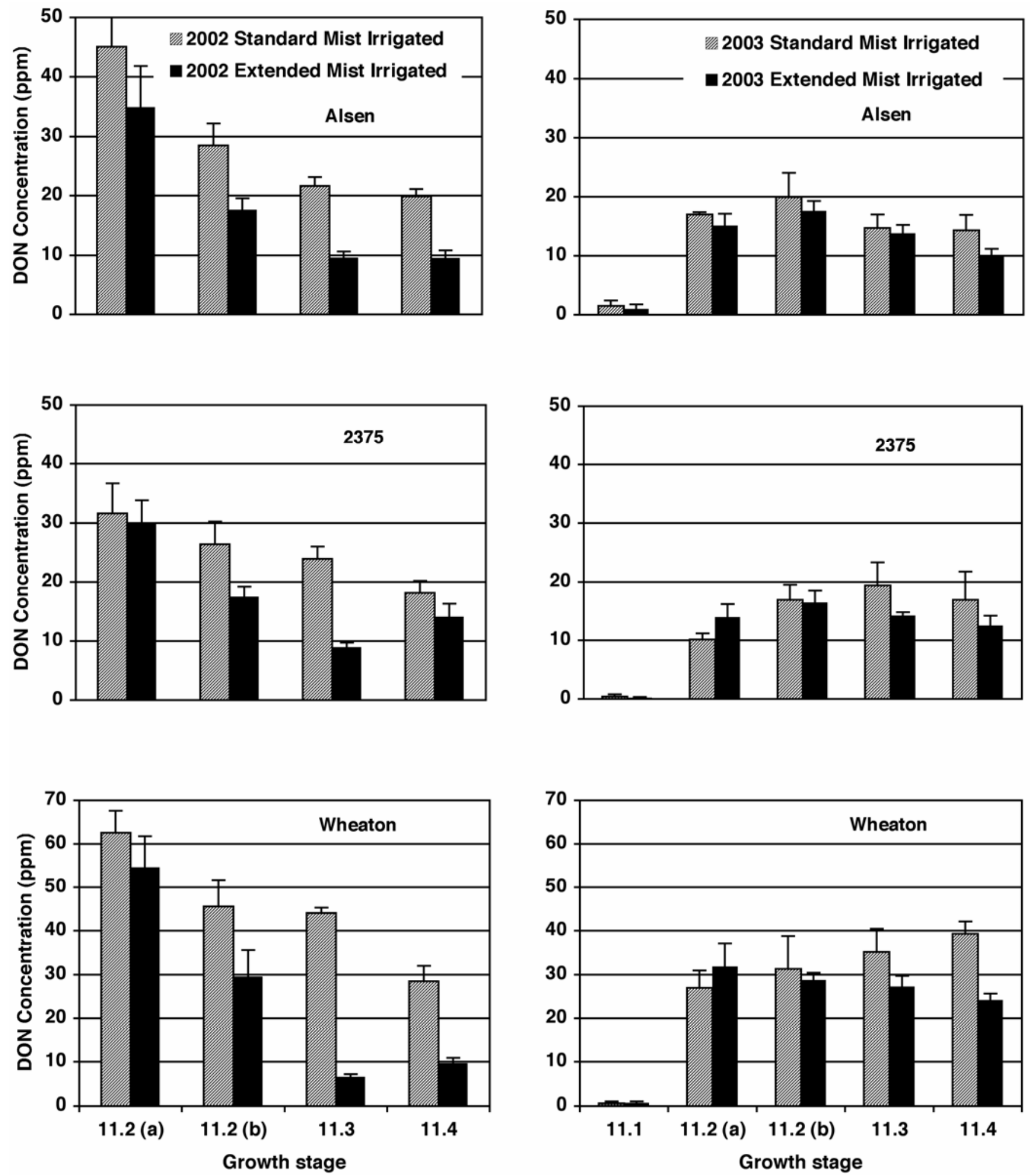

Fig. 3. Deoxynivalenol (DON) concentration in kernels of wheat cultivars Alsen, 2375, and Wheaton collected at growth stages 11.2 (a) (early dough), 11.2 (b) (hard dough), 11.3 (kernel hard), and 11.4 (harvest ripe/maturity) in 2002, and 11.1 (early milk), 11.2 (a), 11.2 (b), 11.3, and 11.4 in 2003, following inoculation with a mixture of isolates of Fusarium graminearum (high inoculation concentration, $1.0 \times 10^{5}$ macroconidia/ml) in plots treated either with extended mist irrigation $(3.6 \mathrm{~mm} /$ day) or standard mist irrigation following disease assessment at St. Paul, MN. Error bars indicate standard errors of means. 
intervals. DON concentrations in spikes peaked 42 d.a.i. and declined to harvest. Although the growth stages at the sampling times were not reported, the moisture content in the spikes had decreased to ca. $10 \%$ prior to the peak in DON, indicating that the grain was drying out and was nearing maturity. Decreases in DON concentrations thus appear to have occurred earlier in our study than in the study by Miller and Young. Differences in the timing of the decline in DON concentrations could be due to differing environmental conditions in the studies. Miller and Young conducted their study on winter wheat, which may differ in the length of time from anthesis to maturity than the spring wheat used in our study; the length of time and the environment during that time might have played a role in the accumulation or reduction of DON.

Declines in DON concentration after anthesis also have been reported from observational field situations in corn, wheat, and barley $(29,34,39)$. In noninoculated winter wheat fields in Ontario, Canada, DON concentrations decreased over the sampling period (39); however, the researchers did not note at which growth stages their samples were collected. Decreasing DON concentrations also were observed in 1 year of a 2-year study of noninoculated barley fields in North Dakota (34).

Hooker et al. (13) developed a model for the prediction of DON concentrations in harvested grain based on weather variables pre- and post-heading. Data from our study neither support nor directly contradict their findings. The model of Hooker et al. (13) identified rainfall periods around heading as being very important in determining final DON concentrations. This model, however, did not include any rainfall events beyond 10 d.a.i., nor did it examine the role of host resistance. The results of our study show that excess moisture after the early dough stage and host resistance are two important factors determining final DON concentrations.

Research by Lemmens et al. (18) also found decreased levels of DON in harvested grain with irrigation in wheat plots inoculated with $F$. culmorum when compared to nonirrigated treatments. Their irrigation treatment started at inoculation (anthesis) and continued only through disease assessment (26 days after anthesis). These results, in combination with our study, suggest that the DON concentration of Fusarium-infected wheat kernels is influenced both by irrigation between anthesis and the assessment of disease (approximately 21 to 26 days after anthesis), and by irrigation between disease assessment and harvest.

Host plant resistance appears to also have a role in the profile of DON accumulation after soft dough. The ability to prevent DON synthesis and promote degradation of DON have been proposed as mechanisms of resistance $(26,28)$. If resis- tance is active, we might expect that DON concentrations would be less relative to that of a susceptible cultivar. The DON concentrations observed in our study were lower and less variable for the FHB resistant cultivar Alsen than for the FHB susceptible cultivar Wheaton under all treatments supporting this argument. These results were expected, as 'Alsen' is reported to be resistant to moderately resistant to FHB (11) and may also be resistant to DON accumulation. 'Alsen' has resistance to FHB derived from the Chinese wheat 'Sumai 3' and conferred in part by the quantitative trait locus Qfhs.ndsu-3BS. In recent work, Lemmens et al. (20) concluded that resistance to DON is a major factor in the resistance of wheat to FHB and hypothesized that Qfhs.ndsu-3BS either encodes a DON-glucosyltransferase or regulates the expression of such an enzyme which would be capable of detoxifying DON in plant tissues.

Snijders and Krechting (40) reported results similar to those found in our study. They sampled kernels of resistant and susceptible cultivars, inoculated with $F$. culmorum, twice between inoculation and harvest, and measured DON concentrations. In a sampling 4 weeks after inoculation, DON levels were higher in the susceptible cultivar than in the resistant. At a second sampling time (8 weeks after inoculation), DON concentrations were lower than for the first sample in the susceptible cultivar, but remained steady or increased slightly in the resistant cultivar. Their DON accumulation pattern results are similar to some of those found in our study under lower disease pressure (Fig. 2 and 3,2003), where DON concentrations in most cases initially increased in susceptible cultivars and then decreased, and DON concentrations in resistant cultivars in most cases were lower at the initial sampling time and remained lower than the DON levels in the susceptible cultivar. However, resistance to DON accumulation may be affected by the severity of FHB infection. In the higher FHB severity field season (2002) at the high inoculum concentration (Fig. 3), DON concentrations were high for both 'Alsen' and '2375'. These data indicate that strong disease pressure may overcome the host plant's resistance.

Our study shows that by increasing the inoculum concentration, we were able to increase the intensity of disease and the level of DON in all three spring wheat cultivars. Our ability to manipulate artificial FHB epidemics in screening nurseries may prove to be quite valuable when higher levels of disease pressure become necessary to accurately select among germplasm types with improved levels of resistance. Further research is warranted to establish how artificial FHB epidemics may be more readily manipulated to achieve a specific range of severities.
DON is not considered to be important in the initial infection of wheat by Fusarium, but the toxin is reported to be important for the growth and spread of the fungus through host tissues $(8,33)$. Once tissue is fully colonized by Fusarium, however, the role of DON may be reduced, and thus the production of DON may peak during the establishment of the fungus in host tissues and fall off once the tissue is fully colonized. A reduction in DON production following colonization of host tissues is supported by recent studies of the expression of genes involved in mycotoxin production in Fusarium examined at different stages of infection of wheat spikes (Frances Trail, personal communication).

Individual spikes of wheat may be infected with a single isolate or multiple isolates of $F$. graminearum (52). Wheat spikes are subject to attack from a number of different fungal and bacterial pathogens, and both living and senescent wheat tissues support an array of microbes that compete for space and nutrients. Thus it may be possible that $F$. graminearum produces DON and other mycotoxins to overcome active host plant defense responses and colonize spike tissues in preference to other pathogens or saprophytes of wheat tissues. Once $F$. graminearum is established in the tissues, DON production may no longer be necessary. It may also be that there are interactions between the isolates of $F$. graminearum infecting the spike, which may limit the DON production by the interacting isolates.

Previous research has indicated that correlations between FHB severity and DON concentrations in harvested grain can be significant but variable from year to year and among locations in a given year $(21,22,41,44)$. In our study, it appears that FHB severity was a better predictor of DON at all stages tested than VSK, although our correlation of 0.65 between VSK and DON was lower than correlations of $r=0.897$ and $r=0.908$ in 1993 and 1994, respectively, reported by Jones and Mirocha (15). One result from our study that did not fit the generalization that FHB severity is a better predictor of DON than VSK was the cultivar Wheaton under the low inoculum concentration treatment. It showed distinctly different levels of FHB severity in 2002 (35.6\%) and 2003 $(11.5 \%)$; however, the levels of DON were almost identical in both years (Fig. 2). Since the FHB severity in Wheaton in 2002 was more than three times that in 2003, we would have expected the DON concentration to be much higher in 2002 than in 2003. These results indicate that yet other factors may influence DON accumulation in Fusarium-infected wheat. These might include the direct effect of light and temperature on the fungus, or indirect effects such as growth stage of the wheat at the time of infection or soil water and nutrient availability, which determine 
overall plant health and response to infection. Evaluation for level of DON concentration should be conducted independently of evaluation for severity of FHB symptoms. In this study, and as previously reported $(24,25)$, environment strongly influenced DON accumulation. Further investigations into the role of environment on DON accumulation throughout the growing season are warranted. Such studies might examine data for DON accumulation following infection of wheat at different growth stages in a range of environments. Further, the sampling intervals could be reduced to improve our understanding of the accumulation and reduction of DON concentrations in various infected tissues over time. These insights into influences on DON accumulation in Fusarium-infected wheat spikes should aid us in reducing the contamination of wheat with Fusarium-produced mycotoxins.

\section{ACKNOWLEDGMENTS}

We thank Yanhong Dong for the deoxynivalenol analyses and Karen J. Wennberg and Amar M. Elakkad for their technical assistance in conducting this research. The financial support of the Minnesota Small Grains Initiative is gratefully acknowledged.

\section{LITERATURE CITED}

1. Abramson, D., Clear, R. M., and Nowicki, T. W. 1987. Fusarium species and trichothecene mycotoxins in suspect samples of 1985 Manitoba wheat. Can. J. Plant Sci. 67:611-619.

2. Bai, G.-H., and Shaner, G. 1994. Scab of wheat: Prospects for control. Plant Dis. 78:760-766.

3. Bechtel, D. B., Kaleikau, L. A., Gaines, R. L., and Seitz, L. M. 1985. The effects of Fusarium graminearum infection on wheat kernels. Cereal Chem. 62:191-197.

4. Campbell, K. A. G., and Lipps, P. E. 1998. Allocation or resources: Sources of variation in Fusarium head blight screening nurseries. Phytopathology 88:1078-1086.

5. Desjardins, A. E., Proctor, R. H., Bai, G., McCormick, S. P., Shaner, G., Buechley, G., and Hohn, T. M. 1996. Reduced virulence of trichothecene-nonproducing mutants of Gibberella zeae in wheat field tests. Mol. PlantMicrobe Interact. 9:775-781.

6. Dill-Macky, R. 2003. Inoculation methods and evaluation of Fusarium head blight resistance in wheat. Pages 184-210 in: Fusarium Head Blight of Wheat and Barley. K. J. Leonard and W. R. Bushnell, eds. American Phytopathological Society, St. Paul, MN.

7. Dill-Macky, R., Evans, C. K., and Culler, M. D. 2001. Manipulating artificial epidemics of Fusarium head blight in wheat with inoculum concentration. Page 113 in: Proc. 2001 National Fusarium Head Blight Forum. S. M. Canty, J. Lewis, L. Siler, and R. W. Ward, eds. Kinko's, Okemos, MI.

8. Eudes, F., Comeau, A., Rioux, S., and Collin, J. 2001. Impact of trichothecenes on Fusarium head blight [Fusarium graminearum] development in spring wheat (Triticum aestivum). Can. J. Plant Pathol. 23:318-322.

9. Evans, C. K., Xie, W., Dill-Macky, R., and Mirocha, C. J. 2000. Biosynthesis of deoxynivalenol in spikelets of barley inoculated with macroconidia of Fusarium graminearum. Plant Dis. 84:654-660.

10. Fernando, W. G. D., Miller, J. D., Seaman, W. L., Seifert, K., and Paulitz, T. C. 2000. Daily and seasonal dynamics of airborne spores of
Fusarium graminearum and other Fusarium species sampled over wheat plots. Can. J. Bot. 78:497-505.

11. Frohberg, R. C., Stack, R. W., Olson, T., Miller, J. D., and Mergoum, M. 2006. Registration of 'Alsen' wheat. Crop Sci. 46:23112312.

12. Gomez, K. A., and Gomez, A. A. 1984. Statistical Procedures for Agricultural Research, 2nd ed. John Wiley \& Sons, New York.

13. Hooker, D. C., Schaafsma, W., and TamburicIlincic, L. 2002. Using weather variables preand post-heading to predict deoxynivalenol content in winter wheat. Plant Dis. 86:611619.

14. Inglis, D. A., and Maloy, O. C. 1983. Scab caused by Gibberella zeae occurring on irrigated wheat in eastern Washington. Plant Dis. 67:827-828.

15. Jones, R. K., and Mirocha, C. J. 1999. Quality parameters in small grains from Minnesota affected by Fusarium head blight. Plant Dis. 83:506-511.

16. Kang, Z., and Buchenauer, H. 1999. Immunocytochemical localization of Fusarium toxins in infected wheat spikes by Fusarium culmorum. Physiol. Mol. Plant Pathol. 55:275288.

17. Kuiper-Goodman, T. 1994. Prevention of human mycotoxicoses through risk assessment and risk management. Pages 439-469 in: Mycotoxins in Grain. Compounds other than Aflatoxin. J. D. Miller and H. L. Trenholm, eds. Eagan Press, St. Paul, MN

18. Lemmens, M., Buerstmayr, H., Krska, R., Schuhmacher, R., Grausgruber, H., and Ruckenbauer, P. 2004. The effect of inoculation treatment and long-term application of moisture on Fusarium head blight symptoms and deoxynivalenol contamination in wheat grains. Eur. J. Plant Pathol. 110:299-308

19. Lemmens, M., Josephs, S., Schuhmacher, R., Grausgruber, H., Buerstmayr, H., Ruckenbauer, P., Neuhold, G., Fidesser, M., and Krska, R. 1997. Head blight (Fusarium spp.) on wheat: Investigations on the relationship between disease symptoms and mycotoxin content. Cereal Res. Comm. 25:459-465.

20. Lemmens, M., Scholz, U., Berthiller, F., Dall'Asta, C., Koutnik, A., Schuhmacher, R., Adam, G., Buerstmayr, H., Mesterhazy, A., Krska, R., and Ruckenbauer, P. 2005. The ability to detoxify the mycotoxin deoxynivalenol colocalizes with a major quantitative trait locus for Fusarium head blight resistance in wheat. Mol. Plant-Microbe Interact. 18:1318-1324.

21. Liu, W., Langseth, W., Skinnes, H., Elen, O. N., and Sundheim, L. 1997. Comparison of visual head blight ratings, seed infection levels, and deoxynivalenol production for assessment of resistance in cereals inoculated with Fusarium culmorum. Eur. J. Plant Pathol. 103:589-595.

22. Love, G. R., and Seitz, L. M. 1987. Effects of location and cultivar on Fusarium head blight (scab) in wheat from Kansas in 1982 and 1983. Cereal Chem. 64:124-128.

23. McMullen, M., Jones, R., and Gallenberg, D. 1997. Scab of wheat and barley: A re-emerging disease of devastating impact. Plant Dis. 81:1340-1348

24. Mesterhazy, A., Bartok, T., Mirocha, C. G., and Komoroczy, R. 1999. Nature of wheat resistance to Fusarium head blight and the role of deoxynivalenol for breeding. Plant Breed. 118:97-110.

25. Miedaner, T., Reinbrecht, C., Lauber, U., Schollenberger, M., and Geiger, H. H. 2001. Effects of genotype and genotype-environment interaction on deoxynivalenol accumulation and resistance to Fusarium head blight in rye, triticale and wheat. Plant Breed. 120:97-105.

26. Miller, J. D., and Arnison, P. G. 1986. Degra- dation of deoxynivalenol by suspension cultures of the Fusarium head blight resistant cultivar Frontana. Can. J. Plant Pathol. 8:147-150.

27. Miller, J. D., and Young, J. C. 1985. Deoxynivalenol in an experimental Fusarium graminearum infection of wheat. Can. J. Plant Pathol. 7:132-134.

28. Miller, J. D., Young, J. C., and Sampson, D. R. 1985. Deoxynivalenol and Fusarium head blight resistance in spring cereals. Phytopathol. Z. 113:359-367.

29. Miller, J. D., Young, J. C., and Trenholm, H. L. 1983. Fusarium toxins in field corn. I. Time course of fungal growth and production of deoxynivalenol and other mycotoxins. Can. J. Bot. 61:3080-3087.

30. Mirocha, C. J., Kolaczkowski, E., Xie, W., and Jelen, H. 1998. Analysis of deoxynivalenol and its derivatives (batch and single kernel) using gas chromatography/mass spectrometry. J. Agric. Food Chem. 46:1414-1418.

31. Neish, G. A., Farnworth, E. R., and Cohen, H. 1982. Zearalenone and trichothecene production by some Fusarium species associated with Canadian grains. Can. J. Plant Pathol. 4:191194.

32. Paulitz, T. C. 1996. Diurnal releases of ascospores by Gibberella zeae in inoculated wheat plots. Plant Dis. 80:674-678.

33. Proctor, R. H., Hohn, T. M., and McCormick, S. P. 1995. Reduced virulence of Gibberella zeae caused by disruption of a trichothecene toxin biosynthetic gene. Mol. Plant-Microbe Interact. 8:593-601.

34. Prom, L. K., Horsely, R. D., Steffenson, B. J., and Schwarz, P. B. 1999. Development of Fusarium head blight and accumulation of deoxynivalenol in barley sampled at different growth stages. J. Am. Soc. Brew. Chem. 57:60 63.

35. Pugh, G. W., Johann, H., and Dickson, J. G. 1933. Factors affecting infection of wheat heads by Gibberella saubinetii. J. Agric. Res. 46:771-797.

36. Salas, B., Steffenson, B. J., Casper, H. H Tacke, B., Prom, L. K., Fetch, T. G., Jr., and Schwarz, P. B. 1999. Fusarium species pathogenic to barley and their associated mycotoxins. Plant Dis. 83:667-674.

37. Savard, M. E., Sinha, R. C., Seaman, W. L., and Fedak, G. 2000. Sequential distribution of the mycotoxin deoxynivalenol in wheat spikes after inoculation with Fusarium graminearum. Can. J. Plant Pathol. 22:280-285.

38. Schroeder, H. W., and Christensen, J. J. 1963 Factors affecting resistance of wheat to scab caused by Gibberella zeae. Phytopathology 53:831-838.

39. Scott, P. M., Nelson, K., Kanhere, S. R., Karpinski, K. F., Hayward, S., Neish, G. A., and Teich, A. H. 1984. Decline in deoxynivalenol (vomitoxin) concentrations in 1983 Ontario winter wheat before harvest. Appl. Environ. Microbiol. 48:884-886.

40. Snijders, C. H. A., and Krechting, C. F. 1992. Inhibition of deoxynivalenol translocation and fungal colonization in Fusarium head blight resistant wheat. Can. J. Bot. 70:1570-1576.

41. Snijders, C. H. A., and Perkowski, J. 1990 Effects of head blight caused by Fusarium culmorum on toxin content and weight of wheat kernels. Phytopathology 80:566-570.

42. Sutton, J. C. 1982. Epidemiology of wheat head blight and maize ear rot caused by Fusarium graminearum. Can. J. Plant Pathol. 4:195-209.

43. Tacke, B. K., and Casper, H. H. 1996. Rapid column cleanup with GC/ECD detection of deoxynivalenol in wheat, barley and malt. J. Int. Assoc. Off. Anal. Chem. 79:472-475.

44. Teich, A. H., Shugar, L., and Smid, A. 1987. Soft white winter wheat cultivar resistant to scab and deoxynivalenol accumulation. Cereal Res. Comm. 15:109-114. 
45. Tuite, J., Shaner, G., and Everson, R. J. 1990. Wheat scab in soft red winter wheat in Indiana in 1986 and its relation to some quality measurements. Plant Dis. 74:959-962.

46. Ueno, Y. 1987. Trichothecenes in Food. Pages 123-148 in: Mycotoxins in Food. P. Krogh, ed. Academic Press, London.

47. Wilcoxson, R. D., Busch, R. H., and Ozman, E. A. 1992. Fusarium head blight resistance in spring wheat cultivars. Plant Dis. 76:658-661.

48. Wilcoxson, R. D., Kommedahl, T., Ozman, E.
A., and Windels, C. E. 1988. Occurrence of Fusarium species in scabby wheat from Minnesota and their pathogenicity to wheat. Phytopathology 78:586-589.

49. Windels, C. E. 2000. Economic and social impacts of Fusarium head blight: Changing farms and rural communities in the northern Great Plains. Phytopathology 90:17-21.

50. Wong, L. S. L., Abramson, D., Tekauz, A., Leisle, D., and McKenzie, R. I. H. 1995. Pathogenicity and mycotoxin production of
Fusarium species causing head blight in wheat cultivars varying in resistance. Can. J. Plant Sci. 75:261-267.

51. Zadoks, J. C., Chang, T. T., and Konzak, C. F. 1974. A decimal code for the growth stages of cereals. Weed Res. 14:415-421.

52. Zeller, K. A., Bowden, R. L., and Leslie, J. F. 2003. Diversity of epidemic populations of Gibberella zeae from small quadrats in Kansas and North Dakota. Phytopathology 93:874 880 . 